\title{
LA RELACIÓN ENTRE CAPITAL INTELECTUAL HUMANO Y CAPACIDAD DE INNOVACIÓN EN EL SECTOR PÚBLICO
}

\section{THE RELATIONSHIP BETWEEN HUMAN INTELECTUAL CAPITAL AND INNOVATION CAPACITY IN THE PUBLIC SECTOR}

\author{
Rodrigo Belmonte da Silva \\ Eje de Gestión y Negocio \\ Instituto Federal Farroupilha \\ São Vicente do Sul, Rio Grande do Sul, Brasil \\ rodrigo.silva@iffarroupilha.edu.br
}

\author{
Carlos Maria Fernandez Jardón \\ Departamento de Economia Aplicada \\ Universidad de Vigo, Vigo, Espana \\ IDLAB, National Research University Higher School of \\ Economics Perm, Russian Federation \\ cjardon@uvigo.es
}

Fecha de recepción: 16/12/2020 - Fecha de aprobación: 20/01/2021

DOI: https://doi.org/10.36995/j.visiondefuturo.2021.25.02R.004.es

\section{RESUMEN}

El sector público debe responder con velocidad e innovaciones a las demandas de los ciudadanos. Esta capacidad puede evitar muchas muertes, como es en el caso de la pandemia de Covid-19. El presente estudio tiene como objetivo general identificar la influencia del capital intelectual humano en la capacidad de innovación del sector público. Para ello, fueron entrevistados 158 servidores públicos que ocupan cargos de liderazgo en la administración pública municipal de la ciudad de América Latina (Santiago - Rio Grande do Sul - Brasil). La técnica estadística utilizada fue el análisis factorial exploratorio y, mediante el análisis de los principales componentes, fueron extraídos dos factores del capital humano: la formación y las habilidades, los valores y las actitudes, además de los constructos de la capacidad de innovación: servicios y procesos, organizacional e institucional. El estudio comprobó la hipótesis de que todos los factores de capital humano se relacionan positivos, significativa y directamente con las dimensiones de la capacidad de innovación. El índice más relevante de correlación fue identificado entre los variables valores y actitudes y la capacidad de servicios y procesos. Por lo tanto, el estudio de caso comprobó la influencia positiva, directa y significativa del capital intelectual humano con la capacidad de innovar del sector público, específicamente, en la administración pública local/municipal en cuestión.

PALABRAS CLAVE: Capital intelectual en el sector público; Capital humano; Capacidad de innovación en el sector público; Innovación en el sector público; Gestión del conocimiento.

\footnotetext{
"Visión de Futuro" Año 18, Volumen No 25 N², Julio - Diciembre 2021 - Pág 114 - 136 URL de la Revista: http://visiondefuturo.fce.unam.edu.ar/index.php/visiondefuturo/index URL del Documento: https://visiondefuturo.fce.unam.edu.ar/index.php/visiondefuturo/issue/view/21 ISSN 1668 - 8708 - Versión en Línea 


\section{ABSTRACT}

The public sector must respond to the citizens' demands with speed and innovation. This ability can prevent many deaths, as in the case with the Covid-19 pandemic. The present study has the general objective of identifying the influence of human intellectual capital on the public sector capacity to innovate. To this end, 158 public servants, who occupy leadership positions in the municipal public administration of the city of Latin America (Santiago - Rio Grande do Sul - Brazil), were interviewed. The statistical technique used was exploratory factor analysis and, through the analysis of the main components, two human capital factors were extracted: training and skills, values and attitudes, in addition to the constructs of innovation capacity: services and processes, organizational and institutional. The study tested the hypothesis that all human capital factors are positively, significantly and directly related to the dimensions of innovation capacity. The most relevant index of correlation was identified among the variables: values and attitudes, and the capacity of services and processes. Therefore, the case of study verified the positive, direct and significant influence of human intellectual capital with the public sector capacity to innovate, specifically, in the local / municipal public administration in question.

KEY WORDS: Intellectual capital in the public sector; Human capital; The Public sector capacity to innovate; Innovation in the public sector; Knowledge management.

\section{INTRODUCCIÓN}

La gestión del conocimiento posee el potencial de influir, mejorar los procesos y renovar el servicio público, además de constituir um importante capacitador para mantener la eficiencia en todas las áreas (Massaro, Dumay \& Garletti, 2015). En este contexto, las administraciones públicas locales/municipales enfrentan un entorno con problemas complejos (Demircioglu y Audretsch, 2018), tales como: desigualdades sociales, violencia, envejecimiento de la población, grandes epidemias, como la experimentada actualmente con el Covid-19. Para solucionarlos, los gobiernos locales deben responder con innovaciones (Lewis et al., 2018).

El conocimiento, desde este enfoque, es distinguido como Capital Intelectual (Rodrigues et al., 2015) que, en la actualidad, se define como una dinámica integrada de conocimiento, experiencias, propiedad intelectual, practicas organizacionales e informacion (Dumay, 2016) resultante de activos intangibles para crear valor (Bratianu, 2018; lacuzzi et al., 2020). En el sector público, el conocimiento pasó a ser un activo intangible fundamental,

\footnotetext{
"Visión de Futuro" Año 18, Volumen N²5 N², Julio - Diciembre 2021 - Pág 114 - 136 URL de la Revista: http://visiondefuturo.fce.unam.edu.ar/index.php/visiondefuturo/index

URL del Documento: https://visiondefuturo.fce.unam.edu.ar/index.php/visiondefuturo/issue/view/21

ISSN 1668 - 8708 - Versión en Línea

E-mail: revistacientifica@fce.unam.edu.ar
} 
teniendo en cuanta la combinación de algunos de sus elementos que son estudiados y correlacionados con la innovación (Demircioglu y Audretsch, 2018; Lewis et al., 2018).

El capital humano es uno de los elementos del conocimiento, específicamente del capital intelectual (Stewart, 1998). Esta dimensión es definida como "(...) todos los aspectos que involucran las aptitudes y competencias de los servidores públicos, ya que sus conocimientos y habilidades son fundamentales para el alcance de los objetivos de una entidad (Rossi et al., 2016, p.698)". Considerando que el entorno complejo forma parte del día a día del sector público y, en este sentido, la capacidad de promover innovaciones es la premisa para la eficiencia, calidad y valor agregado, la investigación de la correlación entre estos temas toma una proporción relevante.

No obstante, las investigaciones en el sector privado presenten resultados empíricos consistentes entre el capital humano y la innovación (Rodrigues et al., 2009; 2015; Jardón y Martos, 2014; Costa, 2010), no existen registros similares en el sector público. Otros estudios abordaron el conocimiento en el sector público (Lewis et al., 2018; Demircioglu y Audretsch, 2018) e identificaron factores intangibles (calidad del liderazgo, drives de innovación, niveles de comunicación) que impactan positivamente en la capacidad de innovar en gobiernos municipales europeos, tales como: Barcelona, Copenhague y Rotterdam. Sin embargo, la influencia del capital humano sobre estos, es desconocida.

Se considera la existencia de una laguna teórica que debe ser explorada: la influencia de los elementos del capital humano y su relación con la capacidad de innovación, específicos para el sector público, teniendo en cuenta las grandes diferencias entre este sector y el privado (Koch et al., 2005). Además de la relación entre los factores, se espera que la propuesta revele variables cuantitativas, especificas, de capital humano, ampliando los hallazgos de Rossi et al., (2016) y de los ítems de capacidad de innovación propuestos por Lewis et al. (2018) y Demircioglu y Audretsch (2018).

Bajo el punto de vista práctico, la pandemia de Covid-19, por ejemplo, exige de los gobiernos/administraciones locales innovaciones y rapidez en sus prácticas y servicios (Entress et al., 2020; Leslie, 2020). Entress et al. (2020) afirman que el liderazgo en el cuidado de la muerte en los Estados Unidos, por ejemplo, carece de um enfoque centrado en el ser humano. En este sentido, las políticas públicas que promueva innovaciones equitativas y protejan los intereses de los ciudadanos parecen ser premisas en este momento (Leslie, 2020). Luego, la contribución practica del articulo consiste en señalar variables del capital humano que favorezcan las practicas innovadoras de los lideres en la administración pública local, con el objetivo de acelerar la dinámica en el desarrollo de

\footnotetext{
"Visión de Futuro" Año 18, Volumen N²5 N², Julio - Diciembre 2021 - Pág 114 - 136 URL de la Revista: http://visiondefuturo.fce.unam.edu.ar/index.php/visiondefuturo/index

URL del Documento: https://visiondefuturo.fce.unam.edu.ar/index.php/visiondefuturo/issue/view/21

ISSN 1668 - 8708 - Versión en Línea

E-mail: revistacientifica@fce.unam.edu.ar
} 
nuevos/modificados servicios y procesos, nuevos objetivos y métodos organizacionales y, además, la capacidad para innovar en políticas y relaciones institucionales.

Por lo tanto, el objetivo principal del artículo busca responder la siguiente pregunta clave: ¿Influye el capital intelectual humano sobre la capacidad de innovación en el sector público local? ¿Qué componentes del capital humano influyen sobre la capacidad de innovar en el sector público local? El estudio propone analizar el caso específico del municipio de América Latina, que posee un modelo de gestión solido con más de diez años.

Para alcanzar este objetivo, se dividió la metodología en dos momentos: en el primero se propusieron componentes y variables teóricas de capital intelectual humano y la capacidad de innovación. A continuación, se probaron y calcularon las dimensiones y variables, estableciendo correlaciones, probando las hipótesis de investigación para, finalmente, sugerir formas de potenciar tanto el desarrollo del capital humano como la capacidad de innovar en el contexto bajo estudio.

El artículo comienza con una introducción y continúa con una breve revisión teórica y una correlación entre los temas. La revisión se basa en la formulación de las hipótesis de investigación a ser probadas, de acuerdo con los métodos de análisis adoptados. Los datos fueron tabulados y analizados, orientados hacia las respuestas de las hipótesis. Finalmente, se recomiendan caminos prácticos y teóricos sobre los temas, además de señalar las posibilidades de investigaciones futuras.

\section{DESARROLLO}

\section{Capital intelectual humano y capacidad de innovación en el Sector Público}

El capital humano es definido por Ekemam y Okpara (2020) como el stock de competencias, conocimientos y atributos incorporados por las personas en su capacidad de realizar el trabajo. En otras palabras, es el conocimiento que los funcionarios llevan consigo cuando salen del edificio, reflejando sus saberes: conocimientos, habilidades, experiencias (Duff, 2018), que influyen en la innovación.

Hartley (2005) presenta um argumento peculiar en relación al tema en el sector público. El autor defiende que esta esfera considera a la innovación como la novedad en acción, o sea, nuevas ideas que funcionan, en este caso, esta va más allá de una intención, significa una idea puesta en práctica. Este argumento reconoce que poner en práctico lo nuevo depende de cambiar, romper con el pasado, afectar expresivamente el modo de operar la organización, o ir más lejos: transformar su filosofía de existencia. Moore et al., (1997, p.276) defienden este postulado al afirmar que “(..) vale la pena reconocer estos

\footnotetext{
"Visión de Futuro" Año 18, Volumen No 25 N², Julio - Diciembre 2021 - Pág 114 - 136 URL de la Revista: http://visiondefuturo.fce.unam.edu.ar/index.php/visiondefuturo/index

URL del Documento: https://visiondefuturo.fce.unam.edu.ar/index.php/visiondefuturo/issue/view/21

ISSN 1668 - 8708 - Versión en Línea

E-mail: revistacientifica@fce.unam.edu.ar
} 
cambios como innovación, (...) debe ser algo nuevo para la organización, ser lo suficientemente grande, general y durable para afectar significativamente las operaciones o el carácter de la organización".

Por lo tanto, para los objetivos de este trabajo, la definición más indicada de innovación para el sector público se delimita como el proceso de generación e implementación de ideas, nuevas o significativamente modificadas, con vistas a crear valor para la sociedad, ya sea que posean um enfoque interno o externo a la administración pública (European Commission, 2013).

Los investigadores reconocen que existen factores denominados determinantes IsidroFilho, 2017), inductores (Halvorsen et al., 2005; Koch y Hauknes, 2005; Agolla y Lill, 2013) o drives (Lewis et al., 2018) que anteceden a los resultados innovadores, por lo tanto, identificados como capacidad de innovación.

La capacidad de innovación se clasifica como una de las tipologías de la capacidad dinámica (Wang y Ahmed, 2007). El contexto de las capacidades dinámicas está relacionado con la teoría basada en recursos (Penrose, 1959; Williamson, 1975; Teece, 1986; Wernerfelt, 1984), el argumento sostiene que las organizaciones mantienen una ventaja competitiva a través de sus recursos internos. Para Teece et al., (1997), la capacidad dinámica se define como la facultad de renovar las competencias con el fin de alinearse con los cambios del entorno de los negocios. Cuanto más crítico sea el momento y complejos los cambios, mayores serán las necesidades de ciertas respuestas innovadoras.

Existen dos vertientes teóricas de pensamiento sobre las capacidades dinámicas (Meirelles y Camargo, 2014), que están conformadas, asimismo, por la capacidad de innovación (Wang y Ahmed, 2007). La primera, considera los comportamientos, habilidades y capacidades organizacionales (Andreeva y Chaika, 2006; Wang y Ahmed, 2007), y la otra, defiende la capacidad de innovación basada en procesos y rutinas (Zollo y Winter, 2002; Winter; 2003; Dosi et al., 2008). Considerando las especificidades de la administración pública en relación a la heterogeneidad y complejidad de su entorno (Demircioglu e Audretsch, 2018) exigen comportamientos y habilidades diferenciadas. Desde este punto de vista, la definición basada en los conceptos de conceptos de Meirelles y Camargo (2014), Zollo y Winter (2002), Teece et al. (2007), Wang y Ahmed (2007); Lewis et al. (2018) y Demircioglu y Audretsch (2018) se menciona:

"la habilidad/aptitud para desarrollar nuevos servicios, procesos, cambios estratégicos organizacionales e institucionales, con base en el conocimiento, habilidades, rutinas, procesos y mecanismos de aprendizaje y gobernanza de las administraciones públicas locales/municipales".

\footnotetext{
"Visión de Futuro" Año 18, Volumen $N^{\circ} 25$ Nº 2, Julio - Diciembre 2021 - Pág 114 - 136

URL de la Revista: http://visiondefuturo.fce.unam.edu.ar/index.php/visiondefuturo/index

URL del Documento: https://visiondefuturo.fce.unam.edu.ar/index.php/visiondefuturo/issue/view/21

ISSN 1668 - 8708 - Versión en Línea

E-mail: revistacientifica@fce.unam.edu.ar
} 
Algunas evidencias de investigaciones hacen que la relación entre el capital humano y la capacidad de innovar sea prometedora. Demircioglu y Audretsch (2018), por ejemplo, argumentan que los activos intangibles, basados en el conocimiento, son influyentes y determinantes en el desarrollo de las capacidades y de innovaciones complejas en el sector público. Los resultados de su investigación sugieren que el comportamiento innovador y la cooperación de los empleados, además de colaborar con importantes fuentes externas y la capacidad de trabajar en un ambiente complejo, se encuentran positivamente asociados a las innovaciones en el sector público, sugiriendo que las influencias de las innovaciones comprenden los niveles individual, organizacional y relacional.

Por otro lado, Henttonen y Ritala (2016), utilizando el modelo de ecuaciones estructurales de datos de la investigación, indagaron a 595 miembros de una organización publica si compartir el conocimiento a nivel individual (en términos de actitudes, estimación de beneficios, auto eficacia y comportamiento actualizado) afectaba el desempeño individual en el trabajo. Los resultados confirman la hipótesis de que la intención de compartir los conocimientos impacta en el desempeño individual y colectivo.

Recientemente, Jalloh y Ming (2020) confirmaron que la capacitación de las personas, o sea, el entrenamiento y desarrollo, compromiso y remuneración de los empleados sirven como elementos estratégicos que facilitan el desarrollo del capital humano en las organizaciones. Además, descubrieron que las distribuciones adecuadas de las recompensas financieras y no financieras se utilizan como motivación y estimulan a las personas para que liberen su creatividad y perspicacia para el crecimiento sustentable de las organizaciones.

Es en este escenario donde se considera la existencia de una influencia positiva entre el capital humano y la capacidad de innovar en la administración pública local, en este sesgo, las hipótesis que se pretenden comprobar son:

H1_El Capital Humano $(\mathrm{CH})$ influye sobre la Capacidad de Innovación de Servicios y Procesos;

H.2_El Capital Humano $(\mathrm{CH})$ influye sobre la Capacidad de Innovación Organizacional;

H.3_El Capital Humano $(\mathrm{CH})$ influye sobre la Capacidad de Innovación Institucional.

\section{Metodología}

La prueba de las hipótesis fueron realizadas en la Administración Pública Local/Municipal de una ciudad de América Latina, específicamente, en el estado más al sur de Brasil (Santiago - Rio Grande do Sul). En la municipalidad se indagó a los gestores de

\footnotetext{
"Visión de Futuro" Año 18, Volumen No 25 N² 2, Julio - Diciembre 2021 - Pág 114 - 136

URL de la Revista: http://visiondefuturo.fce.unam.edu.ar/index.php/visiondefuturo/index

URL del Documento: https://visiondefuturo.fce.unam.edu.ar/index.php/visiondefuturo/issue/view/21

ISSN 1668 - 8708 - Versión en Línea

E-mail: revistacientifica@fce.unam.edu.ar
} 
las secretarias, departamentos y sectores, entre ellos, a servidores públicos concursados y en régimen temporal, teniendo en cuenta que en Brasil existen otros modelos funcionales.

Las respuestas fueron recopiladas entre los meses de diciembre de 2019 y febrero de 2020 mediante cuestionarios dirigidos y no identificados. Se recibieron 158 devoluciones, que representan una tasa de respuesta del $87,7 \%$. Todas las secretarías municipales respondieron las preguntas y presentaron una tasa de respuesta superior al $75 \%$.

El instrumento de investigación fue preparado en base a estudios teóricos y empíricos sobre los temas: capital intelectual humano e innovación en el sector público, habiendo sido probado, en primer lugar, con um número restringido de servidores para ajustar y valorar las preguntas, además de verificar su claridad y comprensión. La elección de los sujetos de la investigación consideró el grado de liderazgo estratégico y táctico, pues participan directamente de la gestión organizacional y de las políticas públicas, supuestamente, bien informados sobre estas y, sobre todo, acerca del modelo de gestión de la municipalidad y, consecuentemente, sus elecciones estratégicas.

\section{Medidas}

Los constructos fueron medidos a través de um cuestionario con escala de Likert de cinco puntos. Los factores del capital intelectual y de la capacidad de innovación fueron analizados de manera multidimensional, de esta forma, se desarrollaron nuevas escalas para los temas, con base en las investigaciones teóricas y empíricas utilizadas (Rossi et al., 2016; Santos-Rodrigues et al., 2015; Bueno et al., 2011, Jardón y Martos, 2014, Demircioglu· y Audretsch, 2018, Grčić y Samaržija, 2016; Lewis et al., 2018, De Vries et al., 2016).

Las dimensiones del capital intelectual humano relevantes para la capacidad de innovación en el sector público fueron medidas a partir de la organización de los siguientes factores:

- Formación y habilidades - como la representación del conjunto de conocimientos y habilidades necesarias para innovar (Bueno et al., 2011; Rossi et al., 2016).

- Valores y actitudes - como valores personales y actitudes necesarias para la innovación (Bueno et al., 2011, Rossi et al., 2016).

Por otro lado, los constructos que formaron la capacidad de innovación en el sector público se concentran en tres dimensiones:

- Capacidad de innovación de servicios y procesos - capacidad de crear o modificar formas mediante las cuales los servicios o procesos son proyectados o proporcionados a los usuarios (Hartley, 2005; Koch et al., 2005).

\footnotetext{
"Visión de Futuro" Año 18, Volumen No 25 N², Julio - Diciembre 2021 - Pág 114 - 136

URL de la Revista: http://visiondefuturo.fce.unam.edu.ar/index.php/visiondefuturo/index

URL del Documento: https://visiondefuturo.fce.unam.edu.ar/index.php/visiondefuturo/issue/view/21

ISSN 1668 - 8708 - Versión en Línea

E-mail: revistacientifica@fce.unam.edu.ar
} 
- Capacidad de innovación organizacional - capacidad de crear o modificar contextos, metas, conceptos o propósitos (Hartley, 2005; Koch et al., 2005; Halvorsen, 2005) o concebir/cambiar formas organizacionales con la introducción de nuevas técnicas de gestión y/o recompensas, o, asimismo, nuevos métodos de trabajo (Bekkers et al., 2006).

- Capacidad de innovación institucional - se refieren a las transformaciones fundamentales en las relaciones institucionales entre organizaciones, instituciones y otros actores del sector público y, más específicamente, en la administración pública (Bekkers et al., 2006).

\section{Técnicas estadísticas}

El primer paso en relación a los datos fue considerar las respuestas de los servidores temporales, teniendo en cuenta que las respuestas de los servidores efectivos presentan índices más confiables en relación a la normalización de los datos. Otro aspecto es que los servidores efectivos son menos sensibles a los cambios de gobierno, teóricamente imparciales, tomando en cuenta su estabilidad funcional. Con esto, el muestreo fue reducido a 104 encuestas validad.

Para la definición de los constructos de capital intelectual humano y la capacidad de innovación se utilizó el análisis exploratorio mediante el ACP (análisis de componentes principales). Las variables que presentan puntajes inferiores a 0,4 no fueron tomadas en cuenta por no asemejarse con el resto de los ítems. Los factores fueron reducidos para explicar mejor la informacion recopilada, para ello, el método Kaiser con porcentaje superior al $50 \%$ aclaran la varianza total.

La rotación fue utilizada en los ítems de capital humano con la intención de ajustar los originales a los diferentes ejes y organizarlos en factores adecuados. La validación de esta técnica fue lograda mediante el test de Bartlett y el coeficiente de Kaiser-Meyer y Okin (KMO). El coeficiente de Alpha de Cronbach probó y comprobó la consistencia interna del cuestionario, considerando los índices superiores a 0,6 como adecuados (Hair Jr., et al, 2005).

Para comprobar finalmente las hipótesis que motivaron este estudio, se utilizaron las técnicas de regresión lineal que permitieron evaluar y comparar el efecto directo de cada variable independiente sobre la dependiente. El proceso de análisis y tratamiento de los datos, así como la evaluación de estos, se realizó a través del paquete estadístico para las ciencias sociales (IBM SPSS Statistics - versión 22 en portugués).

\footnotetext{
"Visión de Futuro" Año 18, Volumen N²5 N², Julio - Diciembre 2021 - Pág 114 - 136

URL de la Revista: http://visiondefuturo.fce.unam.edu.ar/index.php/visiondefuturo/index

URL del Documento: https://visiondefuturo.fce.unam.edu.ar/index.php/visiondefuturo/issue/view/21

ISSN 1668 - 8708 - Versión en Línea

E-mail: revistacientifica@fce.unam.edu.ar
} 


\section{Resultados empíricos}

El análisis empírico fue realizado en dos etapas: en primer lugar, se investigó la confiabilidad de los datos y el análisis factorial exploratorio, para entonces analizar la correlación entre las variables que componen el capital humano y la capacidad de innovación y responder las hipótesis propuestas.

\section{Confiabilidad}

Los coeficientes de Alpha de Cronbach presentan índices superiores a 0,8, indicando una alta confiabilidad y consistencia de los datos internos (Hair Jr. et al., 2005), de acuerdo con lo demostrado en la tabla 1. Los mismos autores afirman que un nivel aceptable de confiabilidad (superior a 0,7 ) significa que los investigados respondieron las preguntas de manera coherente.

Tabla 1 - Análisis de Confiabilidad - Alpha de Cronbach

\begin{tabular}{lcc}
\hline \multicolumn{1}{c}{ Constructos } & Ítems & Alpha de Cronbach \\
\hline Capital Humano $(\mathrm{CH})$ & 18 &, 862 \\
Capacidad de Innovación $(\mathrm{Cl})$ & 8 &, 934 \\
\hline
\end{tabular}

Fuente: Elaboración propia

\section{Análisis Factorial Exploratorio}

La técnica propone sintetizar la información de un considerable número de variables en una cantidad menor de factores. Para ello, se llevó a cabo el Análisis de Componentes Principales (ACP) con el objetivo de relacionar las variables sin determinar en qué medida son coincidentes los resultados con el modelo propuesto (Rodrigues et al., 2009).

El criterio utilizado para la aplicación estadística fue um CUT-off de 0,30 y que los factores expliquen mínimamente en um $50 \%$ los ítems propuestos. A continuación, los factores fueron distribuidos mediante una rotación Varimax con Kaiser Normalization, obteniendo los constructos y sus respectivas variables.

\section{Capital Intelectual Humano}

La reducción de los datos en todos los ítems de capital humano presentó un índice de KMO (Kaiser-Meyer-Olkin) que sugiere una buena correlación entre las variables (KMO = 0,831). Por otro lado, el test de esfericidad de Bartlett señaló un nivel de significancia de 0,000 que aleja la hipótesis de que la matriz de correlación sea la de identidad $(p<0,01)$. Por lo tanto, la conclusión es que existe correlación entre las variables. Con el aval estadístico de la confiabilidad de los datos, fue posible avanzar y confirmar los resultados específicos

\footnotetext{
"Visión de Futuro" Año 18, Volumen No 25 N² 2, Julio - Diciembre 2021 - Pág 114 - 136

URL de la Revista: http://visiondefuturo.fce.unam.edu.ar/index.php/visiondefuturo/index

URL del Documento: https://visiondefuturo.fce.unam.edu.ar/index.php/visiondefuturo/issue/view/21

ISSN 1668 - 8708 - Versión en Línea

E-mail: revistacientifica@fce.unam.edu.ar
} 
del análisis de los componentes principales, con 11 ítems distribuidos en 2 factores comunes y una variancia explicada de 54,67\% (tabla 2). La distribución logró una convergencia de 3 interacciones, de acuerdo con la rotación Varimax con Normalización de Kaiser.

Tabla 2 - Componentes del Capital Intelectual Humano

\begin{tabular}{|c|c|c|}
\hline Componente & Ítem & $\begin{array}{l}\text { Componente } \\
\text { Rotación } \\
\text { Varimax }\end{array}$ \\
\hline \multirow[b]{4}{*}{ CH1_For_Hab } & $\begin{array}{l}\text { Poseo una alta capacidad de comunicación, y tengo la } \\
\text { intención de compartir mis conocimientos. }\end{array}$ & ,746 \\
\hline & $\begin{array}{l}\text { Me considero altamente cualificado y talentoso, al punto } \\
\text { de ampliar mis responsabilidades. }\end{array}$ & ,717 \\
\hline & $\begin{array}{l}\text { Poseo conocimientos especializados en áreas } \\
\text { específicas, garantizando un desempeño superior. }\end{array}$ & ,716 \\
\hline & & \\
\hline (Formación y & Generalmente soy automotivado para el trabajo. & ,678 \\
\hline \multirow[t]{4}{*}{ Habilidades) } & $\begin{array}{l}\text { Contribuyo efectivamente en la búsqueda de soluciones } \\
\text { creativas y nuevas formas de resolver problemas. }\end{array}$ & ,668 \\
\hline & $\begin{array}{l}\text { Mi formación escolar es compatible con las tareas o } \\
\text { funciones que desempeño. }\end{array}$ & 638 \\
\hline & $\begin{array}{l}\text { Mis desempeños, en los trabajos en equipo, } \\
\text { generalmente, presentan mejores resultados que el } \\
\text { trabajo individual. }\end{array}$ &, 515 \\
\hline & $\begin{array}{l}\text { Siento el claro deseo de servir al público y estoy } \\
\text { satisfecho de pertenecer al cuadro de servidores } \\
\text { municipales. }\end{array}$ & ,847 \\
\hline $\begin{array}{l}\text { CH2_Val_Ati } \\
\text { (Valores y }\end{array}$ & $\begin{array}{l}\text { Contribuyo efectivamente en el trabajo colectivo y } \\
\text { coopero siempre que se me solicita. }\end{array}$ & ,805 \\
\hline \multirow[t]{2}{*}{ Actitudes) } & $\begin{array}{l}\text { Considero que las capacitaciones, cuando se fueron } \\
\text { ofrecidas (cursos o seminarios), resultaron en nuevos } \\
\text { conocimientos para la función que desempeño. }\end{array}$ & ,693 \\
\hline & Me considero cordial y sociable en la atención al público. & ,602 \\
\hline
\end{tabular}

Fuente: Elaboración propia

\footnotetext{
"Visión de Futuro" Año 18, Volumen No 25 No 2, Julio - Diciembre 2021 - Pág 114 - 136

URL de la Revista: http://visiondefuturo.fce.unam.edu.ar/index.php/visiondefuturo/index

URL del Documento: https://visiondefuturo.fce.unam.edu.ar/index.php/visiondefuturo/issue/view/21

ISSN 1668 - 8708 - Versión en Línea

E-mail: revistacientifica@fce.unam.edu.ar
} 


\section{Capacidad de innovación en el Sector Público}

El resultado de la ACP, con los mismos criterios aplicados a los elementos del capital intelectual humano, se constató un índice de KMO (Kaiser-Meyer-Olkin) con una muy buena correlación entre las variables $(\mathrm{KMO}=0,835)$. La esfericidad de Bartlett demostró una significancia de 0,000 que rechaza la hipótesis de que la matriz de correlación sea la de identidad $(p<0,01)$. Por lo tanto, la conclusión es que existe correlación entre las variables del elemento. Considerando que los resultados anteriores confirman la confiabilidad estadística, se avanzó en la descripción de los resultados de los factores extraídos. Estos presentaron 4 ítems, explicados por una variancia del 77\% (tabla 3). La distribución no obtuvo convergencia por el hecho de que existe un componente único.

Tabla 3 - Componentes de Capacidad de innovación de servicios y procesos

\begin{tabular}{ccc}
\hline Componente & Ítem & $\begin{array}{c}\text { Matriz de } \\
\text { Componentes }\end{array}$ \\
\hline & Nuevos/Modificados Servicios Internos &, 883 \\
Cl_Serv.Proc & Nuevos /Modificados Servicios Externos &, 879 \\
(Servicios y Procesos) & Nuevos /Modificados Métodos Internos de Trabajo &, 903 \\
& $\begin{array}{c}\text { Nuevas/Modificadas Formas de Resolver Tareas } \\
\text { Externas }\end{array}$ &, 845 \\
\hline
\end{tabular}

Fuente: Elaboración propia

\section{Capacidad de innovación organizacional}

Al analizar estos ítems, la ACP, con los mismos criterios anteriores, se constató um índice de KMO (Kaiser-Meyer-Olkin) que sugiere una aceptable correlación entre las variables $(\mathrm{KMO}=0,500)$. La esfericidad de Bartlett demostró un nivel de significancia de 0,000 que rechaza la hipótesis de que la matriz de correlación sea la de identidad $(p<0,01)$, comprobando la existencia de correlación entre los ítems. Constatando que los datos anteriores apuntan hacia una confiabilidad estadística, se avanzó en la descripción de los resultados de los factores extraídos, explicados por una variancia del $81 \%$ (tabla 4).

\footnotetext{
"Visión de Futuro" Año 18, Volumen N²5 N², Julio - Diciembre 2021 - Pág 114 - 136 URL de la Revista: http://visiondefuturo.fce.unam.edu.ar/index.php/visiondefuturo/index

URL del Documento: https://visiondefuturo.fce.unam.edu.ar/index.php/visiondefuturo/issue/view/21 
Tabla 4 - Componentes de capacidad de innovación organizacional

\begin{tabular}{|c|c|c|}
\hline Componente & Ítem & $\begin{array}{c}\text { Matriz de } \\
\text { Componentes }\end{array}$ \\
\hline & Nuevos/Modificados Objetivos Estratégicos & ,765 \\
\hline \multicolumn{3}{|c|}{ (1) } \\
\hline \multirow[t]{2}{*}{ (Organizacional) } & Nuevos/Modificados Métodos de Incentivo a los & ,963 \\
\hline & Servidores & \\
\hline
\end{tabular}

Fuente: Elaboración propia

\section{Capacidad de innovación institucional}

La ACP de la variable, con los mismos criterios anteriores, se constató um índice de KMO (Kaiser-Meyer-Olkin) que sugiere una aceptable correlación entre las variables $(\mathrm{KMO}=0,500)$. La esfericidad de Bartlett demostró un nivel de significancia de 0,000 que aleja la hipótesis de que la matriz de correlación sea la de identidad $(p<0,01)$. Por lo tanto, existe correlación entre los dos ítems del elemento. Los resultados de los factores extraídos presentaron dos ítems, explicados mediante una variancia del 89,71\% (tabla 5).

Tabla 5 - Componentes de capacidad de innovación Institucional

\begin{tabular}{clc}
\hline Componente & Ítem & $\begin{array}{c}\text { Matriz de } \\
\text { Componentes }\end{array}$ \\
\hline $\begin{array}{c}\text { Cl_Inst } \\
\text { (Institucional) }\end{array}$ & Cl. 7 - Nuevas/Modificadas Políticas Públicas &, 948 \\
& $\begin{array}{l}\text { Cl. } 8 \text { - Nuevas/Modificadas Relaciones } \\
\text { Institucionales }\end{array}$ &, 946 \\
\hline
\end{tabular}

Fuente: Elaboración propia

\section{Correlaciones}

Para estudiar las relaciones entre el capital intelectual humano y la capacidad de innovación, se llevaron a cabo regresiones entre las variables del capital humano con los factores propuestos de la capacidad de innovación en el sector público. Fueron seleccionados los B's con valores superiores a 0,200. De esta manera, fue posible demostrar la existencia de relaciones robustas entre los constructos y la capacidad de innovación, de acuerdo con lo expuesto en las tablas 6,7 y 8.

\footnotetext{
"Visión de Futuro" Año 18, Volumen $N^{\circ} 25$ N² 2, Julio - Diciembre 2021 - Pág 114 - 136

URL de la Revista: http://visiondefuturo.fce.unam.edu.ar/index.php/visiondefuturo/index

URL del Documento: https://visiondefuturo.fce.unam.edu.ar/index.php/visiondefuturo/issue/view/21

ISSN 1668 - 8708 - Versión en Línea

E-mail: revistacientifica@fce.unam.edu.ar
} 
Tabla 6 - Matriz de Regresión: Capital Humano y Capacidad de Innovación de Servicios y Procesos

\begin{tabular}{|c|c|c|c|c|c|}
\hline \multirow{3}{*}{ Modelo } & & \multicolumn{2}{|c|}{ Coeficientes no estandarizados } & \multirow[b]{3}{*}{$\mathbf{t}$} & \multirow[b]{3}{*}{ Sig. } \\
\hline & & \multicolumn{2}{|r|}{ Desvío } & & \\
\hline & & B & Estándar & & \\
\hline \multirow[t]{2}{*}{1} & CH1_For_Hab & 225 & ,082 & 2,730 & ,007 \\
\hline & CH2_Val_Ati &, 522 & ,082 & 6,333 &, 000 \\
\hline
\end{tabular}

Fuente: Elaboración propia

Los resultados indican que el capital humano se encuentra relacionado positiva, directa y significativamente con la capacidad de innovación de servicios y procesos. El modelo resultante es:

Cl_Serv_Proces $=0,225^{*} \mathrm{CH} 1$ For_Hab $+0,522 * \mathrm{CH} 2$ Val_Ati $+\varepsilon$,

Donde:

Cl_Serv_Proces $=$ Capacidad de Innovación en Productos y Procesos

CH1_For_Hab = Capital Humano1 - Formación y Habilidades

$\mathrm{CH} 2 \_$Val_Ati $=$Capital Humano2 - Valores y Actitudes

$\varepsilon,=$ Erro.

Además, es posible concluir que Formación y Habilidades $(0,225$ sig.007) y los Valores y Actitudes (0,522 sig.000) son variables del capital humano con efectos significativos, positivos y directos en la capacidad de innovación de servicios y procesos.

Tabla 7 - Matriz de Regresión: Capital Humano y Capacidad de Innovación Organizacional

\begin{tabular}{|c|c|c|c|c|c|}
\hline \multirow[b]{2}{*}{ Modelo } & & \multicolumn{2}{|c|}{ Coeficientes no estandarizados } & \multirow[b]{2}{*}{$\mathbf{t}$} & \multirow[b]{2}{*}{ Sig. } \\
\hline & & B & Desvío Estándar & & \\
\hline \multirow[t]{2}{*}{1} & CH1_For_Hab & ,231 & ,088 & 2,616 & 010 \\
\hline & CH2_Val_Ati & ,415 & ,088 & 4,712 & ,000 \\
\hline
\end{tabular}

Fuente: Elaboración propia

Los resultados indican que el capital humano se encuentra relacionado positiva, directa y significativamente con la capacidad de innovación organizacional. El modelo resultante es:

$\mathrm{Cl} \_$Org $=0,231^{\star} \mathrm{CH} 1$ For_Hab $+0,415^{\star} \mathrm{CH2}$ Val_Ati $+\varepsilon$,

Donde:

Cl_Org = Capacidad de Innovación Organizacional

CH1_For_Hab = Capital Humano1 - Formación y Habilidades

CH2_Val_Ati = Capital Humano2 - Valores y Actitudes

$\varepsilon$, , Desvío.

\footnotetext{
"Visión de Futuro" Año 18, Volumen N²5 N², Julio - Diciembre 2021 - Pág 114 - 136

URL de la Revista: http://visiondefuturo.fce.unam.edu.ar/index.php/visiondefuturo/index

URL del Documento: https://visiondefuturo.fce.unam.edu.ar/index.php/visiondefuturo/issue/view/21

ISSN 1668 - 8708 - Versión en Línea

E-mail: revistacientifica@fce.unam.edu.ar
} 
Los índices aclaran que la Formación y Habilidades (0,231 sig.010) y los Valores y Actitudes (0,415 sig.000) son variables del capital humano con efectos significativos, directos y positivos en la capacidad de innovación organizacional.

Tabla 8 - Matriz de Regresión: Capital Humano y Capacidad de Innovación Institucional

\begin{tabular}{cccccc}
\hline \multirow{2}{*}{} & & \multicolumn{3}{c}{ Coeficientes no estandarizados } & \\
\cline { 3 - 4 } Modelo & & B & Desvío & & \\
\cline { 3 - 5 } 1 & Estándar & t & Sig. \\
\hline & CH1_For_Hab &, 284 &, 082 & 3,475 &, 001 \\
& CH2_Val_Ati &, 496 &, 082 & 6,062 &, 000 \\
\hline
\end{tabular}

Fuente: Elaboración propia

Los resultados indican que el capital humano se encuentra relacionado positiva, directa y significativamente con la capacidad de innovación institucional. El modelo resultante es:

Cl_Inst $=0,284^{*} \mathrm{CH} 1$ For_Hab $+0,496^{*} \mathrm{CH} 2$ Val_Ati $+\varepsilon$,

Donde:

Cl_Inst = Capacidad de Innovación Organizacional

CH1_For_Hab = Capital Humano1 - Formación y Habilidades

CH2_Val_Ati = Capital Humano2 - Valores y Actitudes

$\varepsilon$, , Desvío.

Los índices aclaran que la Formación y Habilidades (0,284 sig.001) y los Valores y Actitudes (0,496 sig.000) son variables del capital humano con efectos significativos, directos y positivos en la capacidad de innovación Institucional. Las conclusiones definitivas se encuentran ilustradas en la figura 1.

\footnotetext{
"Visión de Futuro" Año 18, Volumen No 25 No 2, Julio - Diciembre 2021 - Pág 114 - 136

URL de la Revista: http://visiondefuturo.fce.unam.edu.ar/index.php/visiondefuturo/index

URL del Documento: https://visiondefuturo.fce.unam.edu.ar/index.php/visiondefuturo/issue/view/21

ISSN 1668 - 8708 - Versión en Línea

E-mail: revistacientifica@fce.unam.edu.ar
} 


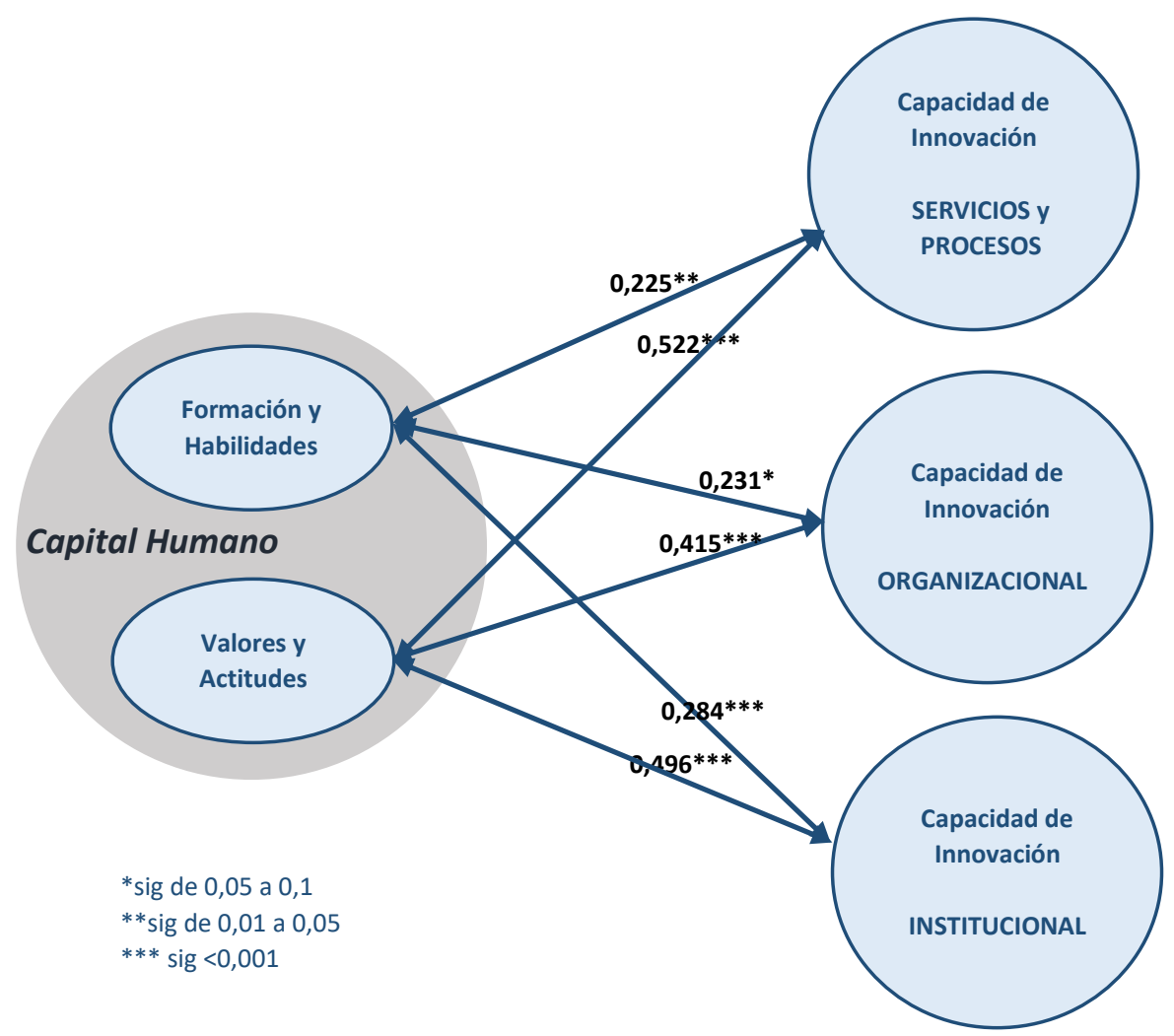

Figura 1 - Relación entre Capital Intelectual Humano y Capacidad de Innovación en el Sector Público Fuente: Elaboración propia

Se puede concluir que el capital intelectual humano influye positiva, significativa y directamente en la capacidad de innovación en el sector público, dentro del contexto investigado, de acuerdo a lo expuesto en la tabla 9 . Se confirman todas las hipótesis.

Tabla 9 - Prueba de Hipótesis

\begin{tabular}{cc}
\hline Hipótesis & Situación \\
\hline H1. El Capital Humano $(\mathrm{CH})$ influye sobre la Capacidad de & CONFIRMADA \\
Innovación de Servicios y Procesos; & Positiva \\
H.2 El Capital Humano $(\mathrm{CH})$ influye sobre la Capacidad de & Directa \\
Innovación Organizacional; & CONFIRMADA \\
& Positiva \\
H.3 El Capital Humano $(\mathrm{CH})$ influye sobre la Capacidad de & Significativa \\
Innovación Institucional. & Directa \\
\hline
\end{tabular}

Fuente: Elaboración propia

\footnotetext{
"Visión de Futuro" Año 18, Volumen No 25 No 2, Julio - Diciembre 2021 - Pág 114 - 136

URL de la Revista: http://visiondefuturo.fce.unam.edu.ar/index.php/visiondefuturo/index

URL del Documento: https://visiondefuturo.fce.unam.edu.ar/index.php/visiondefuturo/issue/view/21

ISSN 1668 - 8708 - Versión en Línea

E-mail: revistacientifica@fce.unam.edu.ar
} 


\section{H1. El Capital Humano (CH) influye sobre la capacidad de innovación de los servicios y procesos}

La hipótesis 1 fue confirma y se demostró que ambos factores: Formación y Habilidades (CH1_For_Hab) y Valores y Actitudes (CH2_Val_Ati) se encuentran asociados a la capacidad de innovación en los servicios y procesos, en el sector público bajo estudio. Específicamente en relación a la formación (educación forma) los hallazgos de Pasamar et al. (2019) fundamentan sus estudios en el presupuesto de que la educación formal de los individuos es directamente proporcional al nivel de desarrollo humano y del desempeño individual. En este contexto, sus descubrimientos señalan que el capital humano es un mediador entre los liderazgos y aprendizajes, funcionando como un fuerte enlace entre ellos. Además, mencionan que: "los individuos que nos mas abiertos a nuevas experiencias y a los riesgos han demostrado contribuir más con el desarrollo de ideas radicales y poseen características tales como la motivación, perfil educativo, profesional histórico y habilidades que pueden influir en la generación e implementación de ideas que lleven a la innovación" (Pasamar et al, 2019. p.30).

La confirmación de la hipótesis contribuye con la propuesta del factor Formación y Habilidades, pues aglutina dos conceptos ampliamente explorados en el contexto del capital humano en el área privada. Al juntar ambos temas, el argumento resultante del factor defiende que la formación escolar, o sea, el conocimiento explicito en el sector público necesita complementarse con las habilidades adquiridas con la experiencia en la función pública, teniendo en cuenta la legalidad constitucional exigida en la administración pública. Entonces, se define la Formación y la Habilidad como: conocimientos formales y know-how para la eficiente ejecución de la función pública.

Por otro lado, de la misma manera, el factor Valores y Actitudes demostró especificidad cuando se encuentra relacionado con el sector público, teniendo en cuenta los principios de la administración pública en relación a la moral, impersonalidad, publicidad y eficiencia que se exigen al servidor, además de iniciativa propia, plena comprensión y voluntad en la ejecución de la practica pública. Por lo tanto, se define el factor Valores y Actitudes como: convicciones, conductas y comportamientos legal y moralmente adecuados para la ejecución de la función pública.

\section{H.2 El Capital Humano (CH) influye sobre la capacidad de innovación organizacional}

La hipótesis 2, de la misma manera, fue confirmada. O sea, Formación y Habilidades (CH1_For_Hab) y Valores y Actitudes ( $\mathrm{CH} 2$ _Val_Ati) se encuentran asociadas a la capacidad de innovación organizacional en el sector público bajo estudio. Nonaka y 
Takeuchi (2008) afirman que la escolaridad, los conocimientos específicos, las habilidades del saber hacer, solamente serán traducidas en capacidad de innovación cuando sean transformadas en conocimiento organizacional. Esta afirmación parece aclarar la correlación entre el factor Formación y Habilidades con la capacidad de innovación organizacional. Será el capital humano el que resulte de conocimientos colectivos y transformándose en nuevos conceptos, nuevas técnicas organizacionales o nuevos métodos de trabajo (Hartley, 2005; Koch et al., 2005; Halvorsen, 2005; Beckkers et al., 2006).

La mayor contribución en la organización de esta hipótesis se encuentra en la configuración del factor Capacidad de innovación organizacional. Mientras que el sector privado comprende los drives de innovación presentes en la mencionada capacidad, tales como liderazgo, cultura, rutinas y procesos (Lewis et al., 2018), se señalan dos aspectos fundamentales desde el punto de vista de este trabajo, para acelerar el proceso de innovación y estimular colectivamente a los servidores: objetivos estratégicos nuevos y/o modificados y sus lineamientos con incentivos para los servidores. Esta propuesta metodológica fue confirmada con una relación positiva con los factores de capital humano, configurando la necesidad de alineación entre la estrategia y los incentivos, en el caso bajo estudio.

\section{H.3 El Capital Humano (CH) influye sobre la capacidad de innovación institucional}

La hipótesis 3 también fue confirmada. Esto significa afirmar que los factores Formación y Habilidades ( $\mathrm{CH}$ 1_For_Hab) y Valores y Actitudes ( $\mathrm{CH} 2$ _Val_Ati) se encuentran asociados a la capacidad de innovación institucional. Estos resultados señalan que los servidores líderes demuestran un alto índice de satisfacción con el trabajo y una especie de orgullo y, consecuentemente, compromiso con el servicio público municipal. El compromiso y la motivación por servir son instrumentos que garantizan un desempeño eficaz en la organización y, como consecuencia, se encuentran relacionados directamente con la creación de un entorno innovador de productos y procesos (Easa y Orra, 2019; Ekemam y Okpara, 2020). Estas cuestiones estimulan un clima de trabajo favorable para que la gestión local proporcione apoyo para la formación de comités internos, un importante activo creativo capaz de fermentar la gestión participativa y crear/modificar servicios, procesos y políticas públicas locales en pro de la mejora de la calidad de vida de los ciudadanos.

La configuración del factor Capacidad de innovación institucional fue una contribución teórica metodológica nueva, comparada con otras investigaciones en el sector público, teniendo en cuenta que no fue identificada ninguna otra dimensión con este nombre, a pesar

\footnotetext{
"Visión de Futuro" Año 18, Volumen No 25 N 2, Julio - Diciembre 2021 - Pág 114 - 136

URL de la Revista: http://visiondefuturo.fce.unam.edu.ar/index.php/visiondefuturo/index

URL del Documento: https://visiondefuturo.fce.unam.edu.ar/index.php/visiondefuturo/issue/view/21

ISSN 1668 - 8708 - Versión en Línea

E-mail: revistacientifica@fce.unam.edu.ar
} 
de la existencia de la dimensión innovación institucional (Beckkers et al., 2006). La correlación positiva con las dimensiones del capital humano constituye un hallazgo para estimular la innovación en el sector público, pues la interacción entre los gestores y los órganos de investigación, universidades, poder político, comunidad en general, además de permitir innovaciones en los servicios y procesos, también es una posibilidad real de creación de innovaciones en las políticas públicas.

\section{CONCLUSIONES}

En síntesis, el estudio confirma la cuestión central de la investigación: afirma que el capital humano influye (positiva, significativa y directamente) sobre la capacidad de innovación (servicios y procesos, organización e institucional) en el sector público estudiado. Además de sugerir posibles contribuciones teóricas prometedoras en dos aéreas: la literatura sobre el capital intelectual e innovación en el sector público y, en la práctica, la identificación de los ítems que influyen directamente sobre la capacidad de innovar, donde la administración pública local puede potenciar sus competencias para cumplir de manera más eficiente las demandas sociales de la comunidad.

La primera contribución se relaciona con la importancia del capital humano como elemento del capital intelectual, considerando que las investigaciones en el sector privado lo señalan como la principal dimensión de este último (Rodrigues et al., 2009; Bontis y Girardi, 2000). El estudio confirmó la importancia del elemento también en el sector público, además de presentar los constructos: formación y habilidades ( $\mathrm{CH} 1$ _For_Hab) y los valores y actitudes ( $\left.\mathrm{CH} 2 \_V a l \_A t i\right)$ como factores exploratorios consistentes para futuras investigaciones.

En relación a la contribución con la bibliografía de capacidad de innovación, se presentan tres factores, de acuerdo con la literatura estudiada: la capacidad de innovación en servicios y procesos (Cl_Serv_Proces), organizacional (Cl_Org) e institucional (Cl_Inst). Además, a través del análisis de los componentes principales, los constructos pretendieron agrupar teóricamente el contenido útil para estudiar el caso, con posibilidades de ampliar y comprender otras tipologías, como ser la capacidad de innovación social, conceptual, entre otras.

Desde el punto de vista práctico, se sugiere la existencia de otros campos para la ampliación del desarrollo humano en la Municipalidad de Santiago. Onah (2015, p.187) afirma que el desarrollo del capital humano se refiere a "(...) un proceso de entrenamiento, educación y otras iniciativas profesionales, con el fin de aumentar el nivel de conocimientos, habilidades, valores y activos sociales de un funcionario, que lo llevará a un nivel masa

\footnotetext{
"Visión de Futuro" Año 18, Volumen No 25 N², Julio - Diciembre 2021 - Pág 114 - 136

URL de la Revista: http://visiondefuturo.fce.unam.edu.ar/index.php/visiondefuturo/index

URL del Documento: https://visiondefuturo.fce.unam.edu.ar/index.php/visiondefuturo/issue/view/21

ISSN 1668 - 8708 - Versión en Línea

E-mail: revistacientifica@fce.unam.edu.ar
} 
elevado de satisfacción y trabajo y, eventualmente, el desempeño de la organización". Por esta razón, a través de un planeamiento consistente, existirán mayores posibilidades de reflejos positivos para el desarrollo del capital humano, de esta manera, los servidores tienden a adquirir nuevas o mejorar sus habilidades para la eficiente ejecución de las funciones para la que fueron contratados (Ekemam y Okpara, 2020).

A pesar de que el estudio ha contribuido señalando posibles caminos que comprueban la influencia del capital humano sobre la capacidad de innovar en el sector público, el modelo puede ser ampliado, pudiendo ser probado en múltiples casos e, inclusive, con una encuesta, en diferentes lugares. La prueba de las mismas hipótesis en distintos escenarios podrá contribuir definitivamente para completar la laguna teórica propuesta, confirmando los descubrimientos aquí detallados.

\section{Agradecimientos}

A los servidores públicos de la Municipalidad de Santiago - Rio Grande do Sul, Brasil.

\section{REFERENCIAS}

Agolla, J. E., \& Van Lill, J. B. (2013). Public Sector Innovation Drivers: A Process Model. Journal of Social Sciences, 34(2), 165-176.

Andreeva, T., \& Chaika, V. (2006). Dynamic capabilities: what they need to be dynamic? [Working Paper, 10 (E)] St. Petersburg State University, São Petersburgo.

Bekkers V., Duivenboden H., M. Thaens (2006), Public Innovation and Communication technology: relevant backgrounds and concepts, in Bekkers V. van Duivenboden $\mathrm{H}$. and Thaens $\mathrm{M}$. (eds), Information and Communication Technology and Public Innovation, IOS Press, Amsterdam/Berlin/Oxford/Tokyo/Washington DC, pp. 3-21.

Bontis, N. y Girardi, J. (2000): "Teaching knowledge management and Intellectual Capital lessons:an empirical examination of Tango simulation", Journal of Technology Management, vol. 20, n. 5/6/7/8, pp. 545-555.

Bratianu, C., (2018) Intellectual capital research and practice: 7 myths and one golden rule. Management \& Marketing. Challenges for the Knowledge Society,13(2), pp.859-879.

Bueno, E.; Real, H. Del.; Fernández, P.; Longo, M.; Merino, C.; Murcia, C.; Salmador, M.P. (2011) Modelo Intellectus: Medición Y Gestión Del Capital

\footnotetext{
"Visión de Futuro" Año 18, Volumen N²5 N², Julio - Diciembre 2021 - Pág 114 - 136 URL de la Revista: http://visiondefuturo.fce.unam.edu.ar/index.php/visiondefuturo/index

URL del Documento: https://visiondefuturo.fce.unam.edu.ar/index.php/visiondefuturo/issue/view/21 
Intelectual. v. Documentos Intellectus.

Costa, R. V (2010): La Influencia del Capital Intelectual em la Innovación de Procuctos: Una Aplicación a Pequeñas y Medianas Empresas Innovadoras de Portugal. Tese de Doutoramento. Universidade de Vigo, Vigo.

De Vries, H.A., Bekkers, V.J.J.M., L.G. (2016). Innovation in the Public Sector: A Systematic Review and Future Research Agenda. Public Administration.

Demircioglu, M., Audretsch D. (2018): Conditions for complex innovations: evidence from public organizations, The Journal of Technology Transfer https://doi.org/10.1007/s10961-018-9701-5.

Dosi, G., Faillo, M. and Marengo, L. (2008) "Organizational Capabilities, Patterns of Knowledge Accumulation and Governance Structures in Business Firms: an Introduction." Organization Studies 29.8-9: 1165-1185.

Duff, A. (2018) Intellectual capital disclosure: evidence from UK accounting firms. Journal of Intellectual Capital Vol. 19 No. 4, pp. 768-786 DOI 10.1108/JIC-062017-0079.

Dumay, J. (2016) A critical reflection on the future of intellectual capital: from reporting to disclosure, Journal of Intellectual Capital, Vol. 17 No. 1, 2016, pp. 168-184, 1469-1930, DOI 10.1108/JIC-08-2015-0072.

Easa, N.F., El Orra, H. (2020) HRM practices and innovation: na empirical systematic review International Journal of Disruptivelnnovation in Government. Emerald Publishing Limited. 2516-4392- DOI 10.1108/IJDIG-11-2019-0005.

Ekemam, H.I., Okpara, C.C. (2020) Human capital development as strategy for effective performance in education sector. African Journal of Social and Behavioural Sciences (AJSBS) Volume 10, Number 1 (2020) ISSN: 2141 209X.

European Commission. (2013) Powering european public sector innovation: towards a new architecture. Report of the Expert Group on Public Sector Innovation, Directorate General for Research and Innovation, Innovation Union, European Commission, Brussels.

Grčić Fabić, M.; Zekić, Z; Samaržija, L. (2016). Implementation of management innovation - a precondition for the development of local government effectiveness: evidence from Croatia. Administratie si Management Public, (27), 7-29.

Hair, J. F.; Babin, B.; Money, A. H. y Samouel, P. (2005), Tradução Lene Belon Ribeiro - Porto Alegre: Bookman.

\footnotetext{
"Visión de Futuro" Año 18, Volumen No 25 No 2, Julio - Diciembre 2021 - Pág 114 - 136 URL de la Revista: http://visiondefuturo.fce.unam.edu.ar/index.php/visiondefuturo/index

URL del Documento: https://visiondefuturo.fce.unam.edu.ar/index.php/visiondefuturo/issue/view/21 
Halvorsen, T. et al. (2005)On the differences between public and private sector innovation. Oslo: Nifu Step. (Publin Report, n. D9).

Hartley, J. (2005). "Innovation in Governance and Public Services: Past and Present", in Public Money \& Management, vol. 25, no. 1, January 2005, pp 27 34.

Henttonen, K., Kianto, A. and Ritala, P. (2016), "Knowledge sharing and individual work performance: an empirical study of a public sector organisation", Journal of Knowledge Management, Vol. 20 No. 4, pp. 749768. https://doi.org/10.1108/JKM-10-2015-0414.

lacuzzi, S., Massaro, M., and Garlatti, A.,2020. Value Creation Through Collective Intelligence: Managing Intellectual Capital. The Electronic Journal of Knowledge Management, 18(1), pp. 68-79, available online at: www.ejkm.com. Isidro-Filho,A. (2017)Inovação no setor público: evidências da gestão pública federal brasileira no período 1999-2014. IN:Inovação no setor público : teoria, tendências e casos no Brasil / organizadores: Pedro Cavalcante ... [et al.]. Brasília : Enap : Ipea.

Jalloh, A.A.A., Ming,F., (2020) Managing Human Capital as a Strategic Source of Sustainable Enterprise Development and Competitive Advantage, International Journal of Management and Humanities (IJMH) ISSN: 2394 - 0913, Volume-5 Issue-1, September.

Jardón, C.M., Martos, M.S. (2014),"Intellectual capital as competitive advantage in emerging clusters in Latin America", Journal of Intellectual Capital, Vol. 13 Iss 4 pp. 462 - 481. http://dx.doi.org/10.1108/14691931211276098.

Koch P., Cunningham P., Schwabsky N., Hauknes J. (2005), Innovation in the Public sector. Summary and policy recommendations. Oslo: Publin Report $n^{\circ}$ D24.

Koch, P.; Hauknes, J. (2005)On innovation in the public sector. Oslo: NIFU STEP.

Lewis, J. M., Ricard, L. M., \& Klijn, E. H. (2018). How innovation drivers, networking and leadership shape public sector innovation capacity. International Review of Administrative Sciences, 84(2), 288-307.

Leslie, D. (2020). Tackling COVID-19 through Responsible AI Innovation: Five Steps in the Right Direction. Harvard Data Science Review. Retrieved from https://hdsr.mitpress.mit.edu/pub/as1p81um

Massaro, M.; Dumay, J.; Garlatti, A. (2015) Public sector knowledge management: a structured literature review. Journal of knowledge management vol. 19 no. 3, pp. 530-558, Emerald Group Publishing Limited, ISSN 1367-3270.

\footnotetext{
"Visión de Futuro" Año 18, Volumen N²5 N², Julio - Diciembre 2021 - Pág 114 - 136 URL de la Revista: http://visiondefuturo.fce.unam.edu.ar/index.php/visiondefuturo/index

URL del Documento: https://visiondefuturo.fce.unam.edu.ar/index.php/visiondefuturo/issue/view/21 
Meirelles, D.S; Bueno Camargo, B.; Álvaro, A. (2014) Capacidades Dinâmicas: O Que São e Como Identificá-las? RAC - Revista de Administração Contemporânea, vol. 18, diciembre, pp. 41-64 Associação Nacional de PósGraduação e Pesquisa em Administração Rio de Janeiro, Brasil.

Moore, M. H., Sparrow, M. and Spelman, W. (1997), Innovation in policing: From production line to jobs shops. In Altchuler, A. and Behn, R. (Eds), Innovation in AmericanGovernment (Brookings Institution, Washington, D.C.).

Nonaka, I. and Takeuchi, H. (1998), The Knowledge Creating Company, Oxford University Press, New York, NY.

Onah, F.O. (2015). Human Resource Management 4th edition. Nsukka: John Jacob"s Classic Publisher.

Pasamar, S., Fernandez, M.D., Navarro, M.D.R.(2018) Human capital: the link between leadership and organizational learning. European Journal of Management and Business Economics Vol. 28 No. 1, pp. 25-51.

Penrose, E. (1959).The Theory of the Growth of the Special Issue, 14, pp. 95-112. Firm. Basil Blackwell, London.

Rodrigues, H.M. da S.S.; Dorrego, P.F.F.; Fernández, C.M.; Fernández, (2009) J. La influencia del capital intelectual en la capacidad de innovación de las empresas del sector de automoción de la Eurorregión Galicia Norte de Portugal. Tese de Doutorado. Universidade de Vigo -Espanha.

Rodrigues, H.S., Jardón,C.M.F., Dorrego,P.F.(2015) Relation between intellectual capital and the product process innovation. Int. J. Knowledge-Based Development, Vol. 6, No. 1.

Rossi, F.M; Francesca C., F.; Bisogno, M. (2016) Intellectual capital in action: evidence from Italian local governments. Journal of Intellectual Capital. Vol. 17 No. 4, 2016. pp. 696-713.

Stewart, T. A. (1998): La nueva riqueza de las organizaciones: el Capital Intelectual, Ediciones Granica, Barcelona.

Teece, D,J.; Pisano, G. and Shuen, A. (1997)Dynamic capabilities and strategic management,Strategic Management Journal, Vol. 18:7, 509-533.

Teece, D. J. (1986). 'Transactions cost economics and and economic organization', Strategic Management the multinational enterprise', Journal of Economic Journal, Winter Special Issue, 12, pp. 75-94. Behavior and Organization, 7, pp. 21-45.

Wang, C. and Ahmed, P. (2007). Dynamic capabilities: a review and research 
agenda.International Journal of Management Reviews, 9, 31-51.

Wernerfelt, B. (1984). 'A resource-based view of the firm Strategic Management Journal, 5 (2), pp. 171-180.

Williamson, O. E. (1975). Markets and Hierarchies. Behavior and Organization, 3, pp. 39-63. Free Press, New York.

Winter, S. G. (2003) The satisficing principle in capability learning. Strategic Management Journal, v. 21, n. 10/11, p. 981-996.

Zollo, M.; Winter, S. G. (2002) Deliberate learning and the evolution of dynamic capabilities. Organization Science, v. 13, n. 3, p. 339-351.

\section{RESUMEN BIBLIOGRÁFICO}

\section{Rodrigo Belmonte da Silva}

Estudiante de doctorado en Administración de la Universidad Nacional de Misiones, AR (UNAM) profesor e investigador el régimen exclusivo del Instituto Federal Farroupilha - Brasil, en las áreas de gestión pública y privada, con énfasis en la gestión estratégica de organizaciones, desarrollo regional e innovación.

\section{Carlos Maria Fernandez Jardón}

Doctor en Economía por la universidad de Navarra y doctor en matemáticas por la universidad de Navarra. MBA executive de Caixanova Business School, Master en gestión del conocimiento por la Universidad Complutense de Madrid. Es Catedrático de Econometría en la Facultad de Ciencias Económicas y Empresariales de la Universidad de Vigo. Ha realizado numerosas investigaciones en el campo de la Economía aplicada y de investigación de mercados. Colaborador en diferentes proyectos de investigación en empresas gallegas y en proyectos de cooperación en Rusia, en Argentina y Paraguay, para el desarrollo de planes estratégicos en cadenas empresariales y proceso de integración productiva transfronteriza. Tiene diferentes publicaciones en temas especializados capital intelectual, innovación y pequeñas empresas en revistas internacionales como el Journal of Intellectual capital, International Journal of Emerging markets, Business Review Quaterly, entre otras.

\footnotetext{
"Visión de Futuro" Año 18, Volumen No 25 N² 2, Julio - Diciembre 2021 - Pág 114 - 136

URL de la Revista: http://visiondefuturo.fce.unam.edu.ar/index.php/visiondefuturo/index

URL del Documento: https://visiondefuturo.fce.unam.edu.ar/index.php/visiondefuturo/issue/view/21

ISSN 1668 - 8708 - Versión en Línea

E-mail: revistacientifica@fce.unam.edu.ar
} 